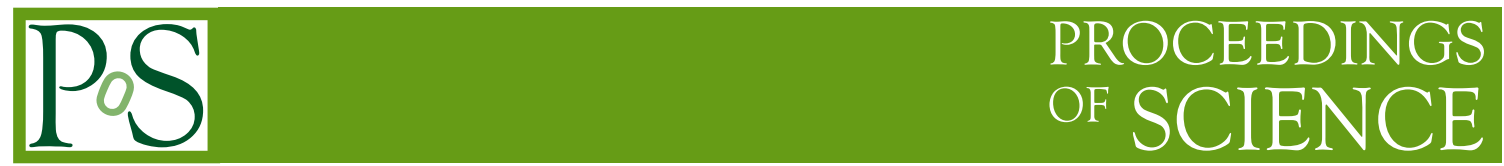

\title{
On the possibility of massless neutrino oscillations
}

\author{
Oleg Kosmachev* \\ VBLHEP JINR \\ E-mail: kos@theor.jinr.ru
}

Qualitative conclusion was obtained about the possibility of massless neutrino oscillations. This result was made in the framework of five fixed assumptions and rigorous consequences on their basis. Such oscillations can arise if particles form a quartet. The quartet is a state, which describes two pairs of particle-antiparticle by means of a single equation. In this case oscillations are determined not by difference of the masses (masses $\mathrm{m}=0$ for each pair), but by the distinction of spin states.

XXI International Baldin Seminar on High Energy Physics Problems September 10-15, 2012

JINR, Dubna, Russia

*Speaker. 


\section{Introduction}

Our approach, proposed for understanding neutrino oscillations, differs markedly from the accepted one. Formally, it is not a negation of generally accepted approach, if only for the obvious reason that here we consider the possibility of massless neutrino oscillations, and not massive ones as it requires by the current approach. Today we do not have evidence that the existence of a massive neutrinos have ruled out the existence of massless ones and vice verse.

What is more, one can show that massive and massless leptons appear on an equal footing in classical works of Dirac [1], Pauli [2], Majorana [3].

Therefore it is interesting to compare massive and massless neutrino oscillations, especially taking into account numerous difficulties and inconsistencies inherent in these area of elementary particle physics. The comparison makes sense and is valuable based on the common principles, which cannot be rejected by any of approaches.

One of the starting points of the prevailing point of view on the oscillations is an assumption, which is called quark-lepton analogy. In fact, it is a "cover" for the gap, in virtue of which lepton sector entirely dropped out of thorough and largely successful theory of unitary symmetry. The analogy as an expression of similarity or similitude with regard to lepton and quarks looks artificial and unjustified.

Indeed, leptons are actually observed particles, whereas quarks are not observed. Adequate description of leptons is impossible out of consistent relativity, while quarks initially are not relativistic structures. That is why leptons found no place among hadrons.

Different ways of relativization of quark model began immediately after its approval. In its time [4], this activity ironically called "the construction of the Babel Tower". This construction is still not finished.

Our approach can be called structural and relativistic. It started with a question of a single description of the lepton sector. In this way, relativity provided a variety of properties, possibility of theoretical identification of each lepton and coverage in the whole lepton sector. As a consequence, it was found that each lepton can be attributed to its own structure. At this stage, it looks as a special, not repeated composition of the wave equation. For the leptons, this is equivalent to the existence of their own quantum numbers.

These and some other arguments allow us to raise the question of possible existence of specific spin oscillation for massless neutrinos. We suppose that the proposed results are necessary prerequisites for spin oscillations. Comparison of our approach with the generally accepted ones shows that they are not interrelated between each other; and each of them is is eligible for further examination until new experimental facts help to make a definite choice among different models.

The great successes of the theory of unitary symmetries indicate that quark structure largely corresponds to the nature of the hadrons. However, the fundamental question of its relation to relativity remains open [5]. Our experience in lepton sector allows us to approach this problem from a new point of view [6]. Explanation of the quark structure of hadrons through relativistic constituents, just as it happened in the lepton sector, eliminates difficulties of the above-mentioned problem. 


\section{Lepton sector}

In the works of last years [7] - [9] we found a possibility to describe a set of massive and massless, charged and neutral, stable and unstable leptons on the basis of Dirac algorithm. The algorithm is necessary and sufficient conditions for formulation of the lepton equations. It was received by means exhaustive group analysis of the Dirac equation.

Irredundant complect of the five initial suppositions, lying in the basis of the algorithm, is following: the equations must be invariant and covariant under homogeneous Lorentz transformations taken into account all four connected components; the equations must be formulated on the base of irreducible representations of the groups, which determine every lepton equation; conservation of four-vector of probability current must be fulfilled, and fourth component of the current must be positively defined; the lepton spin is supposed equal to $1 / 2$; every lepton equation must be reduced to Klein-Fok- Gordon equation (KFG).

All the lepton equations, just like the Dirac equation [1], Pauli [2] equation for the twocomponent massless neutrino and Majorana equation for massive neutrino [3], were obtained without recourse to Euler-Lagrange method. Moreover, a single approach and unified mathematical formalism were developed.

We obtained full and closed set of groups for formulation of wave equations both stable and unstable leptons [8],[9]. The completeness and the closure mean that in the frame of accepted suppositions there is no possibility to obtain additional equations and for their formulation four conjugate components of Lorentz group (i.e. subgroups $d_{\gamma}, f_{\gamma}, b_{\gamma}, c_{\gamma}$ ), or some their combination are sufficient. Concrete sense of all four components is following: group $d_{\gamma}$ realizes proper orthochronous representation; group $f_{\gamma}$ - improper orthochronous or $\langle P\rangle$-conjugate representation; group $b_{\gamma}$ - proper antichronous or $\langle T\rangle$-conjugate representation; group $c_{\gamma}$ - improper antichronous or $\langle P T\rangle=\langle T P\rangle$-conjugate representation.

It was found that all leptons divided into singlets, doublets and quartets, depending on structure of highest order subgroups, . Singlets are the particles that do not have antiparticles. Doublets are associated with equations, which describe one particle and its antiparticle. Finally, quartet equations describe two pairs of the doublets. Each doublet pair is connected to two subgroups. At the same time, each subgroup is identified by its own complete set of quantum numbers, i.e. the set of indexes of this subgroups [10].

In the case of stable lepton groups, maximal subgroups are four subgroups, which realize the four connected components of homogeneous Lorentz group. Here and later infinitesimal form of the group representations is implied and used. All the stable leptons groups have order equal to 32.

As can be seen from the above, classification of leptons (singlets, doublets, quartets) primarily based on the differentiation of the concepts of particles and antiparticles. The criterion of either antiparticle presence or its absence is simple and precise for the stable leptons. The lepton equation describes a particle and antiparticle only if a group of this equation contains $\langle T\rangle$-conjugate connected components. If they are not present, the equation describes the singlet state.

\section{Peculiarities of the massless quartet}

The quartet equation for the neutrino is Pauli equation [2] for two-component massless neu- 
trino, which contains all the four connected components. These four subgroups are related each other through discrete transformations. $\langle P\rangle$ is the inversion of spatial axes, $\langle T\rangle$ is the time conversion and $\langle P T\rangle=\langle T P\rangle$ is the sequential action of the two transformations.

Defining relations for the group of Pauli equation allow for several various formulation. The simplest of them, analogously to the Dirac designations, has the form [7]

$$
\begin{array}{ll}
\gamma_{s} \gamma_{t}+\gamma_{t} \gamma_{s}=2 \delta_{s t}, \gamma_{s}^{2}=1 & (s, t=1,2,3), \\
\gamma_{s} \gamma_{4}-\gamma_{4} \gamma_{s}=0, & (s,=1,2,3), \\
\gamma_{4}^{2}=1 . &
\end{array}
$$

In contrast to Dirac equation, the dimension of $\gamma$-matrices, according to this definition, is $2 \times 2$. As a result, instead of one bispinor solution we obtain four nonequivalent spinor solutions. The four connected components mentioned above are divided in this equation into two pairs, so that within each pair, they are connected with each other by transformation of time reversal $b_{\gamma}=\langle T\rangle d_{\gamma}$ and $c_{\gamma}=\langle T\rangle f_{\gamma}$. The conversation in this case is reduced to the multiplication of three generators of each subgroup $\left(d_{\gamma}, f_{\gamma}\right)$ by imaginary unit $i$.

It is important to note that $\langle T\rangle$-conjugation does not change the main features of spin states. In the case of subgroup $d_{\gamma}$ we can choose any spatial axis as an quantization axis. This is a consequence of the well-known expressions [11] for the raising and lowering operators of 3-rotation group:

$$
\begin{aligned}
& H_{+}=i a_{1}-a_{2}, \\
& H_{-}=i a_{1}+a_{2}, \\
& H_{3}=i a_{3} .
\end{aligned}
$$

Here $a_{1}, a_{2}, a_{3} \equiv a_{1} a_{2}$ are infinitesimal generators of 3-rotation subgroup of $d_{\gamma}$ subgroup. All of them have order equal to four. Therefore, we obtain the equivalence of any spatial axes and value of the first weight number $l_{0}=1 / 2$, which coincides with value of spin $\mathrm{s}=1 / 2$. This fact together with the opportunity to formulate the 4-current of probability allows to speak on the description particles on the basis of subgroups $d_{\gamma}$ and $b_{\gamma}$ with above mentioned spin properties.

We have several other position in a pair of subgroups $f_{\gamma}$ and $c_{\gamma}$. Here the generators of 3rotation subgroup have different orders. One of them has order equal to four, and another one equal to two. Then, according to the equalities (3.2) only one value of $l_{0}$ is equal to $1 / 2$. The two others ones are purely imaginary, i.e. they have no physical meaning. Such an isolated position one of three possible direction is a cause for spin direction along or against the impulse of the particle. The formulation of the 4-vector of current remains possible, but the expression noticeably differs from one in previous pair of subgroups. Therefore, Pauli equation describes the two types of massless particles with different spin properties.

\section{Different types of manifestations of wave properties}

As it is known, the Dirac equation was obtained by decomposition of Klein-Fok-Gordon equation on the product of two factors. Each of them is an expression in space-time derivatives of the first order. In our approach, we obtain immediately the linear equations with respect to the first order derivatives. Therefore reduction to KFG equation is a compulsory and constructive requirement. It reveals the fullness of the wave manifestations of particles and imposes restrictions on 
the particles associated with value of speed $v \leq c$. Here $c$ is the light velocity. In particular, here mutually complementary ratios arise: if $m=0$ than $v=c$ and vice verse, if $m \neq 0$ than $v<c$.

In the general case, formulation of wave the equation is possible in several ways. For example, the Dirac equation contains tree connected components i.e. $d_{\gamma}, f_{\gamma}, b_{\gamma}$. Each of them can be used for explicit writing of equation. Most often it is convenient to write the equation on the basis of proper representation of Lorentz group. It means that $\gamma_{1}, \gamma_{2}, \gamma_{3}$ give rise to subgroup $d_{\gamma}$. In this case, we will call the subgroup $d_{\gamma}$ the facade of equation. The two other subgroups, namely $f_{\gamma}$ and $b_{\gamma}$, are hidden in determining relations, which are well known: $\gamma_{\mu} \gamma_{v}+\gamma_{v} \gamma_{\mu}=2 \delta_{\mu v},(\mu, v=1,2,3,4)$.

In the cited Dirac paper, decomposition of KFG equation was carried out in two identical factors. Shortly and conditionally, this can be written in the following way:

$$
(K F G)=\left(\ldots d_{\gamma} \ldots\right)\left(\ldots d_{\gamma} \ldots\right)
$$

Here, in the right part, each factor is the facade for recording of the Dirac equation on the basis of proper representation of Lorentz group i.e. by means of $d_{\gamma}$ group. The left part is the wave equation. It is suitable for description of any relativistic particles. You need only to take into account the presence or absence of its masses. All information characterizing the electron or positron is lost completely into KFG wave equation. Such transition from two linear equations, describing the identical particles, to the purely wave KFG equation can be interpreted as necessary condition for the interference of identical particles.

It turned out that in addition to Dirac's decomposition of KFG equation, when the two factors are identical expressions, there are other possible combinations of the factors. In particular, the transition to the KFG equation is always available for doublet equations, if factors are $\langle T\rangle$ conjugate between themselves. In fact, it means the recording of one factors for particle and second one for antiparticle. Then it can be written similarly to previous expression:

$$
(K F G)=\left(M_{T}\right)\left(\ldots d_{\gamma} \ldots\right)\left(\ldots b_{\gamma} \ldots\right)
$$

Common multiplier $\left(M_{T}\right)$ appears here in contrast to the expression (4.1). This type of reduction to the KFG equation is necessary condition for annihilation. The equality of (4.1) type is possible for any lepton equation. Their number for each equation is equal to number of connected components of given equation. We will call the two factors as a crossed factors, if they are not identical. In particular, the factors of (4.2) type we will call $\langle T\rangle$-crossed.

As it was already noted, the Pauli equation contains all four connected components. Therefore, an additional opportunities for decomposition of KFG equation on linear factors occurred. If the faced of the quartet equation is chose on the basis of $d_{\gamma}$ subgroup, than equation takes the form proposed by Pauli [2].

All possible transitions between subgroups $d_{\gamma}, b_{\gamma}, f_{\gamma}, c_{\gamma}$ are as follows:

$$
\begin{aligned}
& \langle T\rangle d_{\gamma}=b_{\gamma}, \quad\langle P\rangle d_{\gamma}=f_{\gamma}, \quad\langle P T\rangle d_{\gamma}=c_{\gamma}, \\
& \left\langle T^{-1}\right\rangle b_{\gamma}=d_{\gamma},\langle P\rangle b_{\gamma}=c_{\gamma}, \quad\left\langle T^{-1} P\right\rangle b_{\gamma}=f_{\gamma}, \\
& \left\langle T^{-1}\right\rangle c_{\gamma}=f_{\gamma},\left\langle P^{-1}\right\rangle c_{\gamma}=b_{\gamma},\left\langle T^{-1} P^{-1}\right\rangle c_{\gamma}=d_{\gamma}, \\
& \langle T\rangle f_{\gamma}=c_{\gamma}, \quad\left\langle P^{-1}\right\rangle f_{\gamma}=d_{\gamma},\left\langle P^{-1} T\right\rangle f_{\gamma}=b_{\gamma} .
\end{aligned}
$$


The practical realization of discrete operations is sometimes called the analytical continuation by group parameters. For the operator $\langle T\rangle$ it is reduced to the replacement:

$$
b_{k} \rightarrow b_{k}^{\prime}=i b_{k} \quad(k=1,2,3),
$$

where $b_{k}$ are three generators of transformed group. Each of four subgroups $d_{\gamma}, b_{\gamma}, f_{\gamma}, c_{\gamma}$ has in its structure the 3-rotation subgroup, which is defined by two generators $a_{1}, a_{2}$. Operation $\langle P\rangle$ is reduced to replacement of only one of them, according to the rule:

$$
a_{2} \rightarrow a_{2}^{\prime}=i a_{2} .
$$

Both above-named operations commute with each other; therefore, their successive action looks like this: $\langle P T\rangle=\langle T P\rangle$.

Equations (4.3) and (4.4)-(4.5) allow to get all possible expressions for cross-factors which are linear in the first derivatives. Reduction to KFG for facade $d_{\gamma}, b_{\gamma}$ and their cross-factors $\left(\ldots d_{\gamma} \ldots\right)\left(\ldots b_{\gamma} \ldots\right)$ leads to expressions of the form (4.1) and (4.2).

$$
(K F G)=\left(\ldots d_{\gamma} \ldots\right)\left(\ldots d_{\gamma} \ldots\right) \Rightarrow \partial^{2} / \partial t^{2}=\partial^{2} / \partial x^{2}+\partial^{2} / \partial y^{2}+\partial^{2} / \partial z^{2}
$$

Here and later common multiplier $\left(1 / c^{2}\right)$ before space derivatives is omitted.

Similar, but more simple expressions are obtained for factors $\left(\ldots f_{\gamma} \ldots\right)\left(\ldots f_{\gamma} \ldots\right),\left(\ldots c_{\gamma} \ldots\right)\left(\ldots c_{\gamma} \ldots\right)$ and cross-factor $\left(\ldots f_{\gamma} \ldots\right)\left(\ldots c_{\gamma} \ldots\right)$ :

$$
(K F G)=\left(\ldots c_{\gamma} \ldots\right)\left(\ldots c_{\gamma} \ldots\right) \Rightarrow \partial^{2} / \partial t^{2}=\partial^{2} / \partial z^{2}
$$

Products of $\langle P T\rangle$-conjugate factors such as $\left(\ldots b_{\gamma} \ldots\right)\left(\ldots f_{\gamma} \ldots\right)$ and $\left(\ldots d_{\gamma} \ldots\right)\left(\ldots c_{\gamma} \ldots\right)$ with accuracy up to common multiplier have the same form as (4.7).

Another situation is emerged for purely $\langle P\rangle$-conjugate factors $\left(\ldots d_{\gamma} \ldots\right)\left(\ldots f_{\gamma} \ldots\right)$ and $\left(\ldots b_{\gamma} \ldots\right)\left(\ldots c_{\gamma} \ldots\right)$. Here the reduction to KFG equation leads to it non-standard form:

$$
(K F G) \Rightarrow \quad \partial^{2} / \partial t^{2}=\partial^{2} / \partial z^{2}+2 \sigma_{z} \partial^{2} / \partial z \partial t
$$

Evidently, here information on spin state of initial system is preserved partially. This can be considered as a necessary condition for manifestation of spin oscillations.

\section{Summary}

The study of the massless quartet properties on the basis of general assumptions revealed three types of wave property manifestations. Such manifestations are interference of the identical particles, annihilation between the particles, which are interrelated with each other by $\langle T\rangle$-conjugation operation, and oscillations of particles are connected with each other only by $\langle P\rangle$-conjugation. In each of three cases it is known that the particles are described by means of one equation or another. The distinctive features of the massless quartet of particles are that all the three types of wave manifestations are possible. Therefore, it becomes not only does the origin of their differences become clear, but also their similarities.

Detailing of lepton structures is necessary and important for future applications. In addition, we correct unnatural situation when based on experiments we assign various properties or some quantum numbers to leptons without having specific carriers of those characteristics. They can be named internal properties or symmetries, but the goal is to explain them. 


\section{References}

[1] Dirac P., Proc.Roy. Soc. A vol. 117,610 (1928).

[2] Pauli W., Handbuch der Physik, (Verlag Julius Springer, Berlin) v. 24, p. 226 (1933).

[3] Majorana E., Il Nuovo Cimento v. 14, 171 (1937).

[4] Pais A., Rev. Mod. Phys. 38, 215 , (1966)

[5] O’Raifertaigh L., Phys.Rev 139, B 1052 (1965)

[6] Kosmschev O., Phys. At. Nucl. V. 75, 885 (2012)

[7] Kosmachev O., Representations of the Lorentz group and classification of stable leptons: Preprint JINR, Đ2-2006-6 (Dubna, 2006). (in Russian)

[8] Gusev A, Kosmachev O., Phys. Part. Nucl. Lett. V. 5, 67 (2008).

[9] Kosmachev O., Phys. Part. Nucl. Lett. V. 7, 85 (2010).

[10] Weyl G., The Theory of Groups and Quantum Mechanics,(Dover Publ., 1936;Nauka, Moscow, p.16, 1986).

[11] Gel'fand I., Minlos R., Shapiro Z., Representations of rotation group and Lorentz group (M.:FM, p.193, 1958). (in Russian) 